\title{
Outcrossing and selfing evolution in populations under directional selection
}

\author{
JACQUES L. DAVID*†, YVAN SAVY† \& PHILIPPE BRABANT † \\ †Station de Génétique Végétale, INRA-UPS, Ferme du Moulon, F-91190 Gif sur Yvette, and $\ddagger$ Institut National Agrono- \\ mique Paris-Grignon, 16 rue Claude Bernard, F-75005 Paris, France
}

\begin{abstract}
Many plant species have a mixed mating system using self-pollination and cross-pollination. Such behaviour has no simple evolutionary explanation. When inbreeding depression is low the theory predicts that self-pollination should be fixed. A residual crossing habit exists in practically all selfpollinated species that show low inbreeding depression. As recombination is useful to generate new genotypes it can be valuable when the environmental conditions are not stable.

We used a simulation model to study the dynamics of adaptation with regard to different mixing mating systems. The evolution of a quantitative trait related to fitness has been related to the mating behaviour. In a first approach, all the individuals of a population outcrossed with the same proportion. The comparison between populations differing for their outcrossing rate, their effective size and their initial level of adaptation for new enironments, led us to the conclusion that the smaller the population and the lower its adaptation level, the more valuable outcrossing is. An optimum level of outcrossing has been found for each situation studied. In a second approach, selfing rate was controlled by a polymorphic additional locus. Thus, individuals with different outcrossing behaviours competed within each population. We found that a low level of outcrossing can be maintained and randomly fixed in such conditions, in spite of no inbreeding depression. The outcrossing rate that can be fixed when individuals with different mating behaviour compete is much lower than the optimum outcrossing rate found for the whole population.
\end{abstract}

Keywords: directional selection, evolution, mixed mating system, outcrossing rate, selfing rate, simulation.

\section{Introduction}

In contrast to animals, many plant species can selffertilize. Some of them have a mixed mating behaviour and a portion of their zygotes also comes from outcrossing. The existence of such mixed mating systems has no simple evolutionary explanation. Many authors have dealt with that issue considering the relationships between mating system and inbreeding depression (Fisher, 1941; Nagilaki, 1976; Wells, 1979; Charlesworth, 1980; Lande \& Schemske, 1985). Their models predict that selfing should be complete when inbreeding depression is low and outcrossing should be complete otherwise.

Some mechanisms have then been suggested for the existence of mixed mating: transient situations before fixation of selfing, balance between ecologically reversible conditions favouring selfing, gene flow between

\footnotetext{
*Correspondence.
}

populations preventing this fixation and sampling errors (Schemske \& Lande, 1985). Recent models also deal with the coevolution of selfing and inbreeding depression when the genotypes at the mating system loci are not independent of the depression effect induced by selfing: selfing purges the populations of deleterious alleles making the inbreeding depression decrease (Holsinger, 1988; Charlesworth et al., 1990; Uyenoyama \& Waller, 1991). They describe the evolution of the mating system not only with the relative performance of inbred and outcrossed offspring but also with the positive associations between the mating system and the viability when deleterious mutations occur at the viability loci.

An advantage in recombination for outcrossing in predominantly selfers has also been suggested permitting new adaptation (Maynard-Smith, 1978; Stebbins, 1957 in Lande \& Schemske, 1985; Crow, 1992). Lewontin (1974) demonstrated that an optimum of recombination exists for adaptation in a multilocus 
context. However, that advantage in recombination for outcrossing could be insufficient to lead to stable equilibria with selfing (Lande \& Schemske, 1985).

Nevertheless, in preferential selfing species, for which inbreeding depression is weak, low levels of outcrossing are often found (Allard \& Workman, 1963; Harding \& Tucker, 1965; Kahler et al., 1975; Agren \& Schemske, 1993). This could be grounds for evolutionary mechanisms, and if so, it could be considered as a particular breeding system in response to variable environments. In such conditions, what is the optimum mating strategy for the whole population and what is the maximum level of outcrossing that can actually be maintained as selfing alleles should become rapidly fixed in the absence of inbreeding depression? To answer these questions, we simulated the evolution of ill-adapted populations (not yet adapted to a new environment). We did not try to find stable equilibria between outcrossing and selfing but to understand the dynamics of adaptation with regard to different mixed mating systems. We supposed that fitness is a quantitative trait determined by many loci so that many recombinations are necessary to get the best adapted genotype that does not exist in the initial populations. If the number of loci is too small, the best genotype appears too quickly to visualize the recombination advantage of outcrossing. The selfing rate is controlled by an additional locus that has no direct action on fitness. This could appear unrealistic but it is actually equivalent to the mating modifiers of the previous quoted models. Moreover, there is no inbreeding effect in the model.

First the selfing rate was fixed for all the individuals within a population and we compared the evolution of the selective value of different populations with different selfing rates. This first approach will be later referred to as 'group selection'. We particularly studied the influence of the size and genetic composition of the populations.

In a second part we added within-population polymorphism for the selfing rate to verify if partial outcrossing could be maintained under individual selection. This second approach will be referred to as 'individual selection'.

\section{Materials and methods}

\section{The model}

We developed a computer model very close to those described in breeding or in natural selection studies to simulate quantitative traits (Mani et al., 1990; Hospital \& Chevalet, 1993). The code had been written in FORTRAN 77 and we used the random number generator proposed by Lécuyer (1988).
The model simulates the evolution of a hermaphroditic diploid population of $N$ individuals. The population size is kept to $N$ during all successive generations. Eighty independent loci determine the genotypic fitness. At each locus two different alleles are possible, one favourable and one unfavourable. We assigned the values 2,1 and 0 respectively to the favourable homozygote, heterozygote and unfavourable homozygote. The fitness of a genotype is obtained by summing up the elementary values at its 80 loci so that the worst genotypic value is 0 and the best is 160 .

An 81 st independent locus determines the outcrossing rate ' $t$ '. The effective outcrossing rate of an individual is obtained by summing up the values of its two outcrossing alleles. Thus, an individual whose genotype is [0.01/0.01] at the mating locus, mates 2 per cent of its ovules with out-pollen and self-fertilizes the remaining 98 per cent. Outcrossing is simulated as panmixia.

The program generates the next generation with the previous one following three steps: selection, mating and fertilization. For each individual of the old generation, a relative selective value is computed according to its genotypic value and the mean value of the population. $N$ new individuals are then successively generated. A random number determines the mother according to its relative selective value. According to the selfing rate of this female, a second random number determines if the new individual will proceed from selfing or outcrossing. In the latter case, a last random number determines the father according to its relative selective value. Two gametes are finally created by simulating meiosis between independent genes and are associated to create the new individual.

\section{Definition of the initial populations}

We created starting populations according to three parameters: $N$, the number of individuals in the population, $P$ the mean frequency of the favourable alleles (all the viability loci have the same frequency expectation for the favourable allele), and $t$ the mean outcrossing rate. The genotypes of the first generation were generated by randomly drawing favourable alleles with the probability $P$ in a uniform distribution. All the initial populations were created by simulating panmixia at the 80 viability loci.

We studied two values for $N(100$ and 1000 individuals $)$ and for $P(0.5$ and 0.1$)$. This leads to four parameter sets. They will be quoted as: $(N=1000, P=0.5)$, $(N=1000, P=0.1),(N=100, P=0.5)$ and $(N=100$, $P=0.1)$. In the group selection study, $t$ was fixed for a given population and changed only over simulations. We studied the influence of $t$ varying from 0 to 1 on the evolution of fitness. 
In the individual selection study, one-half of the starting population was supposed homozygote $[0,0]$ at the mating locus and the other half homozygote $[A, A]$. The respective outcrossing rates of the two genotypes were then 0 and $2 A$, the heterozygotes arising in the following generations outcrossed in a proportion $\mathrm{A}$, so that $t$ varied every generation with the frequency of the outcrossing allele. A simulation under individual selectio.. will then be referred to as 0 vs. $2 A$. Three values of A were tested, namely $0.005,0.015$ and 0.025 giving three situations, $(0$ vs. 1 per cent $),(0$ vs. 3 per cent $)$ and $(0$ vs. 5 per cent $)$. To determine the selection influence on the evolution of $t$, each class of mating competition has been studied under selective neutrality and under directional selection. In the neutrality pattern, there was no selective difference between individuals; their fitness was $1 / N$.

For each $(N, P)$ parameter set, we computed at least 30 independent replicates. At each generation we calculated the mean and the variance of the fitness value, the number of polymorphic genes, and especially for the individual selection pattern we recorded the allelic frequencies at the mating locus.

\section{Results}

\section{Group selection}

In all group selection cases, the evolution of fitness over generations strongly varied according to outcrossing. Yet no mating strategy appeared to be the best over the whole range of generations, i.e. the best mating strategy depended on the number of generations after starting. In the first generations high selfing populations increased their fitness the fastest but their rate of improvement rapidly decreased. In the following generations, intermediate outcrossing populations had a better fitness (see Fig. 1 for $(N=1000, P=0.5))$.

At least in large populations, an intermediate outcrossing rate rapidly became the best strategy and remained so until the 500th generations for which fitness is almost maximum. That optimum rate was $t=0.2$ for $(N=1000, P=0.5)$ after the 70th generation (Fig. 1) and $t=0.4$ for $(N=1000, P=0.1)$ after the 180th (Fig. 2). In these large populations, complete panmixia $(t=1.0)$ never provided the best fitness even in the long range. In fact when populations outcrossed at a rate higher than 0.2 in the $(N=1000, P=0.5)$ pattern, their fitness values always remained under that of the optimum population and they only caught up with it asymptotically (Fig. 1).

Figure 3 plots the optimum outcrossing rates against generations and also indicates the mean population fitness obtained with that mating system at this genera-
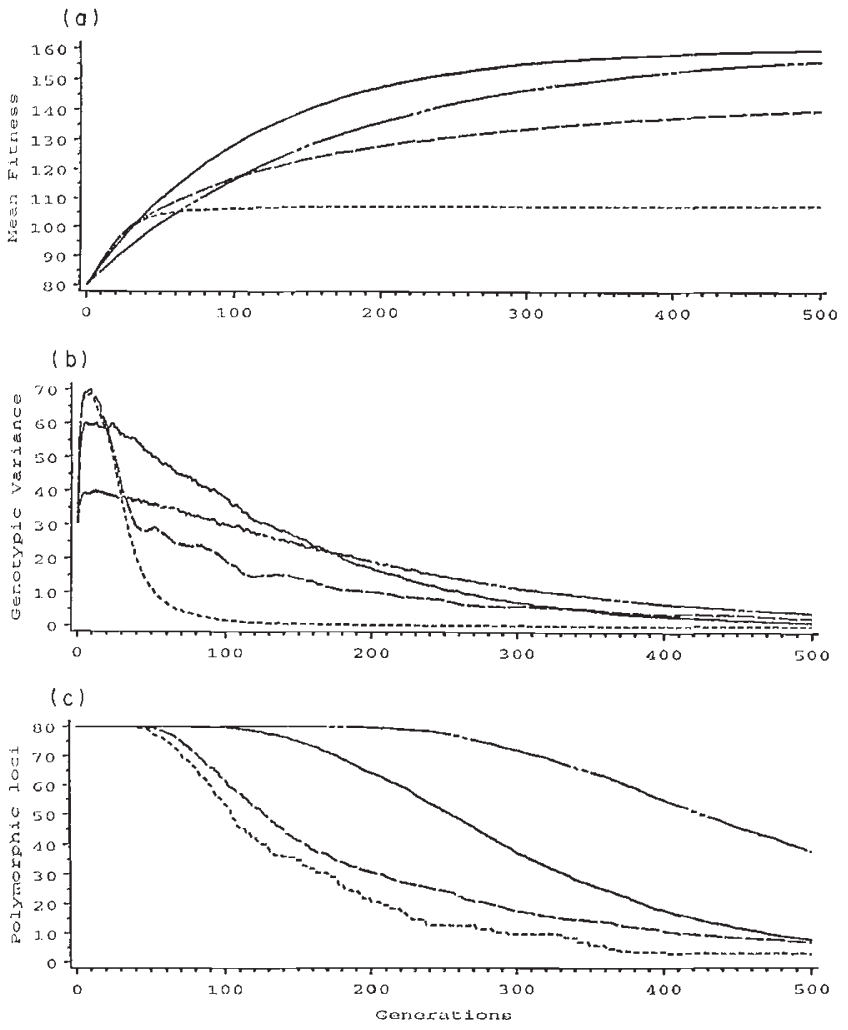

Fig. 1 Evolutions of populations of 1000 individuals under directional selection with four different fixed outcrossing rates, respectively, $t=0.00(\ldots), t=0.01(---), t=20(\square)$ and $t=1.00(\cdot-)$. The mean frequency of the 80 valuable fitness alleles equals 0.5 at generation 0 . Each curve represents the mean of 30 simulations. (a) Evolution of the mean fitness. (b) Evolution of the genotypic variance. (c) Evolution of the polymorphism, defined as the number of remaining polymorphic loci.

tion. The situation in small populations (Fig. 3c, 3d) showed that outcrossing optima are higher than in large populations (Fig. 3a, b) and that no mating strategy remained the best for a long period of time. Nevertheless, for $(N=100, P=0.5)$, the $t=0.5$ strategy remained the best for 150 generations during which fitness increased dramatically. Higher rates and complete panmixia became the best strategies only in late generations when fitness increased very little. The situation was similar for $(N=100, P=0.1)$ : optima were found around $t=0.5-0.7$ during a large period between the 50th and the 250th generations. More than half of the fitness was realized during this period (Fig. 3d).

In large populations, the relative difference in fitness between optimum outcrossing rates and the other rates were higher than in small populations. The maximum difference between optima and complete panmixia reached +10 per cent in the $(N=1000, P=0.5)$ 

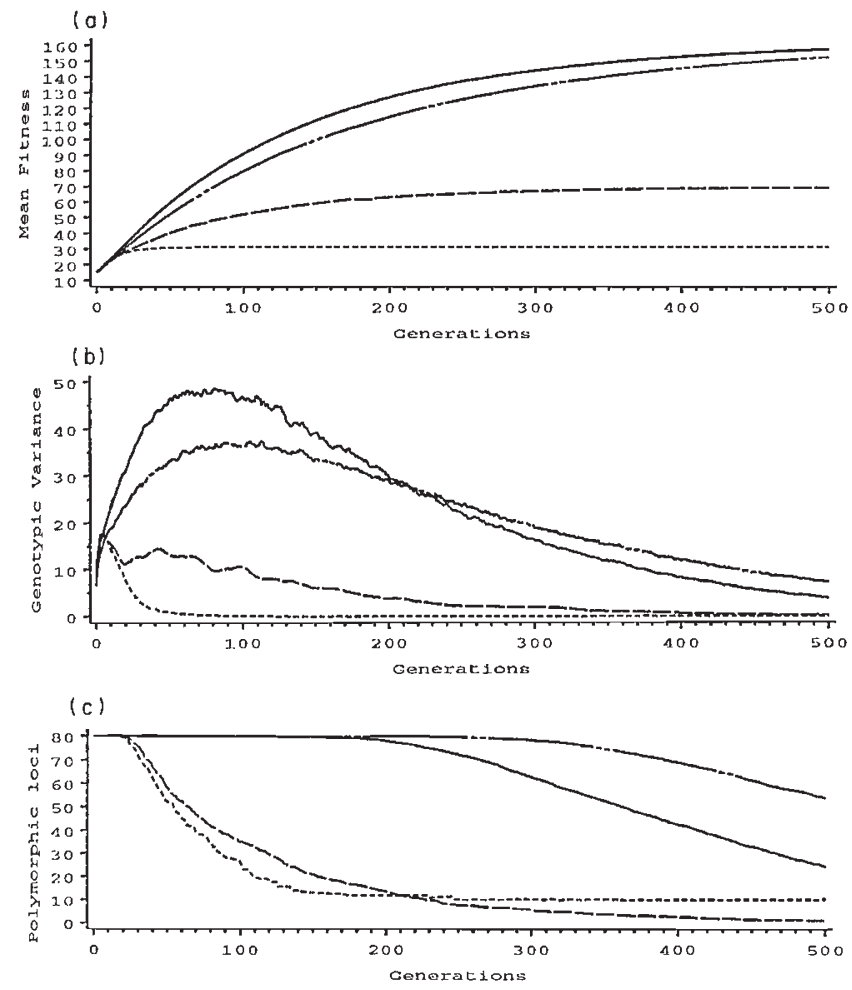

Fig. 2 Evolutions of populations of 1000 individuals under directional selection with four different fixed outcrossing rates, respectively, $t=0.00(\ldots), t=0.01(---), t=0.40(-)$ and $t=1.00(-\cdot \cdot)$. The mean frequency of the 80 valuable fitness alleles equals 0.1 at generation 0 . Each curve represents the mean of 30 simulations. (a) Evolution of the mean fitness. (b) Evolution of the genotypic variance. (c) Evolution of the polymorphism, defined as the number of remaining polymorphic loci.

pattern (Fig. 1) and +12.6 per cent for $(N=1000$, $P=0.1$ ) (Fig. 2) when it was only +3 per cent for $(N=100, P=0.5)$ (curves not presented). The genetic advance was also limited in the long range in small populations (Fig. 3b, c) and could not reach the maximum value of 160 whatever the outcrossing rate.

When favourable alleles were unfrequent, low outcrossing rates provided a better strategy than complete selfing in the long range. For $(N=1000$, $P=0.1$ ) the mean fitness of $t=0.01$ populations at the 500 th generation was +89 per cent higher than that of $t=0.0$ populations, when the same difference was only +26 per cent in the $(N=1000, P=0.5)$ situation (Figs. 1 and 2).

The magnitude and the evolution of the genotypic variability also greatly changed over situations. For the $(N=1000, P=0.5)$ case (Fig. 1b), the genotypic variance increased under complete selfing $(t=0)$ during the first 10 generations as quickly as it decreased afterwards. That first increasing period roughly corresponds to setting up homozygosity in the population. The variance reduction continued until the 100th generation when nearly no more genetic variance was left. For the $t=0.2$ optimum population, the variance decreased slowly after a first increase also due to partial homozygosity. Until the 160th generation it remained over the variance of the panmictic population, which did not benefit from any first fixation increase. This occurred although the number of polymorphic loci was almost always higher in outbred populations compared to more selfed ones (Fig. 1c).

Initial populations had little genotypic variation when $P=0.1$ (Fig. 2b). Under high selfing, the variance increase induced by fixation was small and variances rapidly fell (Fig. 2b). Under intermediate and high outcrossing, genotypic variances increased until the population mean fitness reached 80 , which roughly corresponds to a mean frequency of 0.5 for the fitness alleles. Thanks to partial homozygosity, the largest genetic variance for numerous generations was still obtained with the optimum outcrossing rate (Fig. 2b).

When diminishing the population size, genotypic variances followed roughly the same evolutions as in large populations but maxima were lower and decreases sharper.

\section{Individual selection}

In this section, the mating modifier locus was polymorphic in initial populations.

\section{Neutral conditions}

Under neutral conditions, it was of no interest to vary the initial allelic frequency of fitness alleles as all individuals had the same selective value. So, six different situations were studied (Table 1): $N=1000$ and $N=100$ faced with three different competition patterns ( 0 vs. 1 per cent $),(0$ vs. 3 per cent $)$ and ( 0 vs. 5 per cent).

In large populations $(N=1000$, Table $1, \mathrm{~A})$ none of the 30 simulations led to the fixation of the partial outcrossing allele whatever the competition pattern whereas it sometimes occurred in small populations, especially for the low outcrossing alleles ( 1 per cent and 3 per cent) (Table 1,B). The selfing allele was also fixed more rapidly when it competed with higher partial outcrossing (Table 1,A). Moreover, alleles were fixed sooner in small populations.

\section{Selection}

Twelve situations were studied (Tables 2 and 3) under directional selection for fitness: the six situations, 
(a)

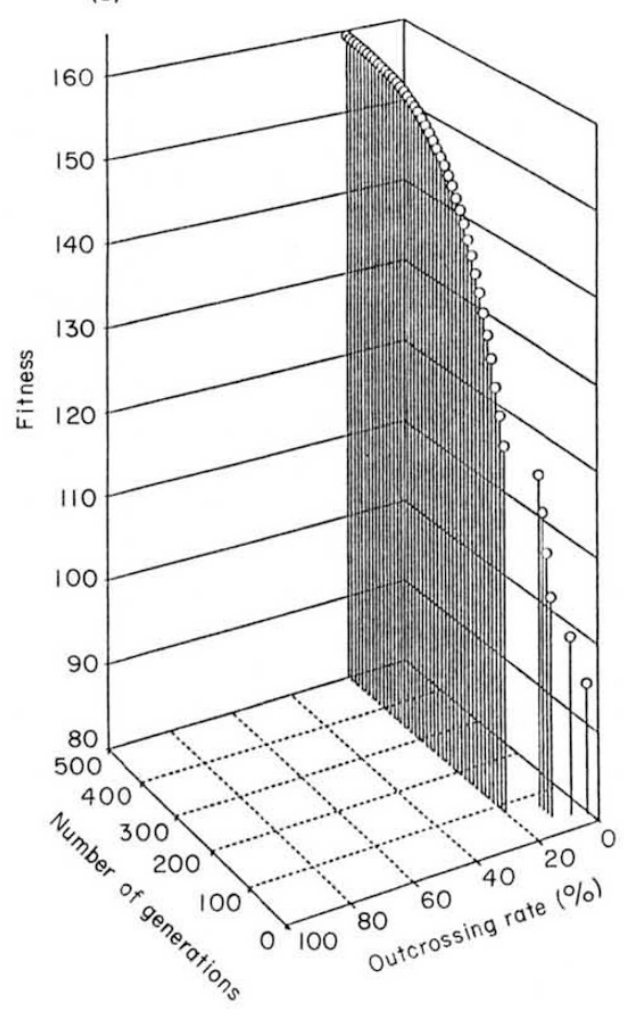

(c)

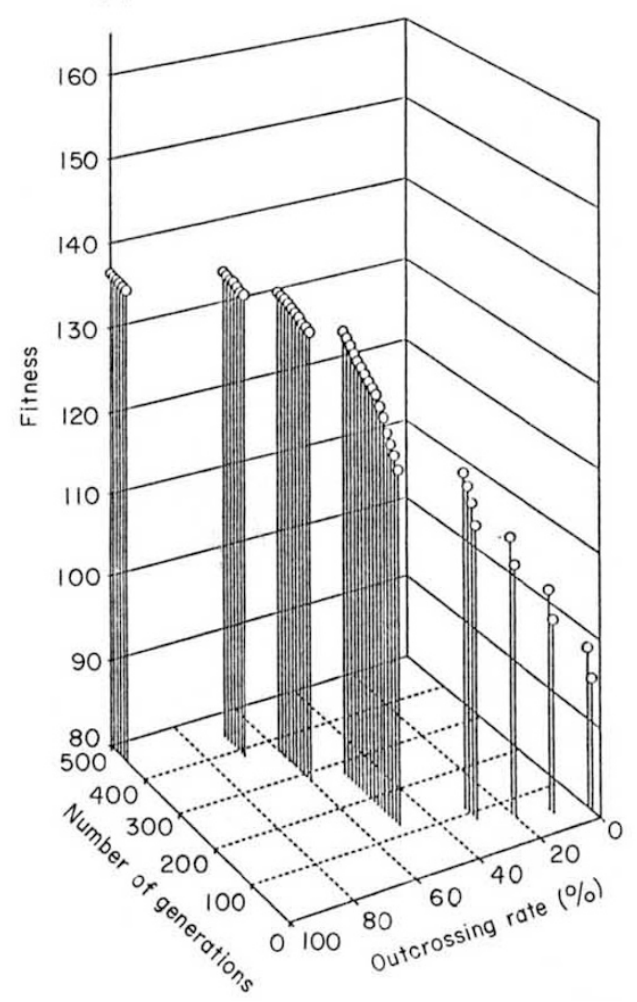

(b)
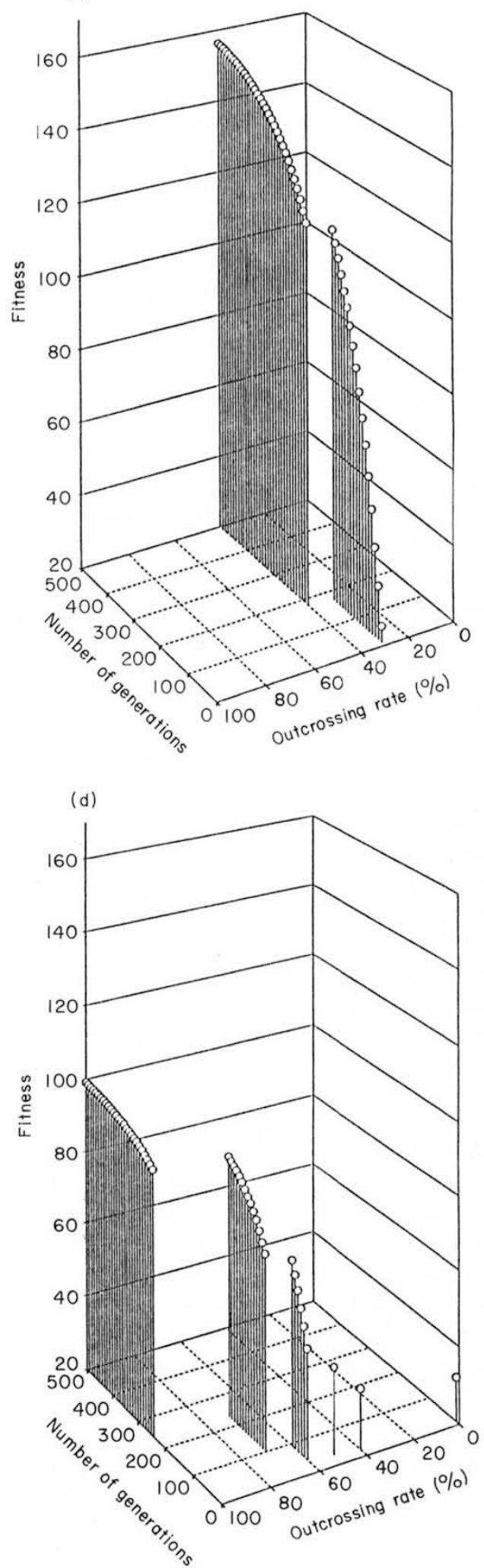

Fig. 3 Best mating strategies that gave the highest fitness level after a given number of generations. Only one generation in every 10 is plotted. A spot represents the mean fitness obtained over 30 independent simulations. The four graphs correspond to four different initial situations. (a) Large populations, $N=1000$, favourable fitness alleles were present in the starting population with a mean frequency of $P=0.5$. (b) Large populations, $N=1000$, favourable fitness alleles were present in the starting population with a mean frequency of $P=0.1$. (c) Small populations, $N=100$, favourable fitness alleles were present in the starting population with a mean frequency $P=0.5$. (d) Small populations, $N=100$, favourable fitness alleles were present in the starting population with a mean frequency of $P=0.1$. 
Table 1 Evolution of outcrossing alleles under neutrality. Number of simulations that either fixed the selfing allele or fixed the outcrossing allele or that still remained polymorphic after a given number of generations. Three different partial outcrossing alleles competed vs. a complete selfing allele, e.g. $(0$ per cent vs. 1 per cent $),(0$ per cent vs. 3 per cent $)$ and $(0$ per cent vs. 5 per cent)

\begin{tabular}{|c|c|c|c|c|c|c|c|c|}
\hline \multirow[b]{2}{*}{$\begin{array}{l}\text { Competition } \\
\text { pattern }\end{array}$} & \multicolumn{4}{|c|}{ A (under neutral conditions) } & \multicolumn{4}{|c|}{$\mathrm{B}$ (under neutral conditions) } \\
\hline & Generation & $\begin{array}{l}\text { Selfing } \\
\text { fixed }\end{array}$ & $\begin{array}{l}\text { Polymorphic } \\
\text { state }\end{array}$ & $\begin{array}{l}\text { Partial } \\
\text { outcrossing } \\
\text { fixed }\end{array}$ & Generation & $\begin{array}{l}\text { Selfing } \\
\text { fixed }\end{array}$ & $\begin{array}{l}\text { Polymorphic } \\
\text { state }\end{array}$ & $\begin{array}{l}\text { Partial } \\
\text { outcrossing } \\
\text { fixed }\end{array}$ \\
\hline$[0,0.005]$ & 50 & 0 & 30 & 0 & 25 & 0 & 28 & 2 \\
\hline \multirow[t]{2}{*}{$(0 \%$ vs. $1 \%)$} & 150 & 2 & 28 & 0 & 50 & 9 & 18 & 3 \\
\hline & 500 & 20 & 10 & 0 & 500 & 23 & 0 & 7 \\
\hline$[0,0.015]$ & 50 & 0 & 30 & 0 & 25 & 0 & 29 & 1 \\
\hline \multirow[t]{2}{*}{$(0 \%$ vs. $3 \%)$} & 150 & 17 & 13 & 0 & 50 & 9 & 19 & 2 \\
\hline & 500 & 30 & 0 & 0 & 500 & 23 & 1 & 6 \\
\hline$[0,0.025]$ & 50 & 0 & 30 & 0 & 25 & 2 & 28 & 0 \\
\hline \multirow[t]{2}{*}{$(0 \%$ vs. $5 \%)$} & 150 & 29 & 1 & 0 & 50 & 9 & 21 & 0 \\
\hline & 500 & 30 & 0 & 0 & 500 & 29 & 0 & 1 \\
\hline
\end{tabular}

A: 1000 individuals per population. Results are given for the 50th, 150th and 500th generations. B: 100 individuals per population. Results are given for the 25 th, 50 th and 500 th generations.

Table 2 Evolution of outcrossing alleles under directional selection in large populations ( $\mathrm{mN}=1000$ individuals). Number of simulations that either fixed the selfing allele or fixed the outcrossing allele or that still remained polymorphic after a given number of generations. Three different partial outcrossing alleles competed vs. a complete selfing allele, e.g. (0 per cent vs. 1 per cent $),(0$ per cent vs. 3 per cent $)$ and $(0$ per cent vs. 5 per cent $)$

\begin{tabular}{|c|c|c|c|c|c|c|c|}
\hline \multirow[b]{3}{*}{$\begin{array}{l}\text { Competition } \\
\text { pattern }\end{array}$} & \multirow[b]{3}{*}{ Generations } & \multicolumn{3}{|c|}{$\mathrm{A}(P=0.5)$} & \multicolumn{3}{|c|}{$\mathrm{B}(P=0.1)$} \\
\hline & & \multicolumn{3}{|c|}{ Under directional selection } & \multicolumn{3}{|c|}{ Under directional selection } \\
\hline & & $\begin{array}{l}\text { Selfing } \\
\text { fixed }\end{array}$ & $\begin{array}{l}\text { Polymorphic } \\
\text { state }\end{array}$ & $\begin{array}{l}\text { Partial } \\
\text { outcrossing } \\
\text { fixed }\end{array}$ & $\begin{array}{l}\text { Selfing } \\
\text { fixed }\end{array}$ & $\begin{array}{l}\text { Polymorphic } \\
\text { state }\end{array}$ & $\begin{array}{l}\text { Partial } \\
\text { outcrossing } \\
\text { fixed }\end{array}$ \\
\hline$[0,0.005]$ & 50 & 3 & 26 & 1 & 5 & 18 & 7 \\
\hline \multirow[t]{2}{*}{$(0 \%$ vs. $1 \%)$} & 150 & 9 & 12 & 9 & 13 & 6 & 11 \\
\hline & 500 & 13 & 0 & 17 & 16 & 1 & 13 \\
\hline$[0,0.015]$ & 50 & 5 & 25 & 0 & 4 & 18 & 8 \\
\hline \multirow[t]{2}{*}{$(0 \%$ vs. $3 \%)$} & 150 & 12 & 15 & 3 & 7 & 9 & 14 \\
\hline & 500 & 21 & 3 & 6 & 10 & 2 & 18 \\
\hline$[0,0.025]$ & 50 & 12 & 18 & 0 & 3 & 21 & 6 \\
\hline \multirow[t]{2}{*}{$(0 \%$ vs. $5 \%)$} & 150 & 19 & 10 & 1 & 8 & 19 & 11 \\
\hline & 500 & 28 & 1 & 1 & 19 & 0 & 11 \\
\hline
\end{tabular}

Results are given for the 50th, 150 th and 500th generations. A: Mean initial frequency of the favourable alleles in fitness was 0.5 . B: Mean initial frequency of the favourable alleles in fitness was 0.1 .

already listed for neutrality, faced with two different values of ' $P$ ' $(P=0.5$ and $P=0.1)$.

Partial outcrossing alleles sometimes became fixed whatever the initial conditions were (Tables 2 and 3).

In large populations, the 1 per cent allele was the most frequently fixed at the 500th generations when $P$ was equal to 0.5 (Table 2,B). We compared the selective value of the different outcrossing alleles with their respective ratio of the number of times when selfing became fixed to the number of times when partial outcrossing became fixed. The $13 / 17$ distribution observed for ( 0 vs. 1 per cent) is significantly different from the $21 / 6$ (chi-squared 1 d.f. $=7.14$ ) and $28 / 1$ (chisquared, 1 d.f. $=19.71)$ distributions observed for $(0 \mathrm{vs.}$ 
Table 3 Evolution of outcrossing alleles under directional selection in small populations ( $\mathrm{mN}=100$ individuals). Number of simulations that either fixed the selfing allele or fixed the outcrossing allele or that still remained polymorphic after a given number of generations. Three different partial outcrossing alleles competed vs. a complete selfing allele, e.g. ( 0 per cent vs. 1 per cent $),(0$ per cent vs. 3 per cent $)$ and $(0$ per cent vs. 5 per cent $)$

\begin{tabular}{|c|c|c|c|c|c|c|c|}
\hline \multirow[b]{3}{*}{$\begin{array}{l}\text { Competition } \\
\text { pattern }\end{array}$} & \multirow[b]{3}{*}{ Generations } & \multicolumn{3}{|c|}{$\mathrm{A}(P=0.5)$} & \multicolumn{3}{|c|}{$\mathrm{B}(P=0.1)$} \\
\hline & & \multicolumn{3}{|c|}{ Under directional selection } & \multicolumn{3}{|c|}{ Under directional selection } \\
\hline & & $\begin{array}{l}\text { Selfing } \\
\text { fixed }\end{array}$ & $\begin{array}{l}\text { Polymorphic } \\
\text { state }\end{array}$ & $\begin{array}{l}\text { Partial } \\
\text { outcrossing } \\
\text { fixed }\end{array}$ & $\begin{array}{l}\text { Selfing } \\
\text { fixed }\end{array}$ & $\begin{array}{l}\text { Polymorphic } \\
\text { state }\end{array}$ & $\begin{array}{l}\text { Partial } \\
\text { outcrossing } \\
\text { fixed }\end{array}$ \\
\hline$[0,0.005]$ & 25 & 7 & 21 & 2 & 22 & 5 & 3 \\
\hline \multirow[t]{2}{*}{$(0 \%$ vs. $1 \%)$} & 50 & 14 & 8 & 8 & 24 & 2 & 4 \\
\hline & 500 & 18 & 0 & 12 & 25 & 0 & 5 \\
\hline$[0,0.015]$ & 25 & 9 & 21 & 0 & 16 & 13 & 1 \\
\hline \multirow[t]{2}{*}{$(0 \%$ vs. $3 \%)$} & 50 & 14 & 14 & 2 & 21 & 4 & 5 \\
\hline & 500 & 22 & 0 & 8 & 22 & 0 & 8 \\
\hline$[0,0.025]$ & 25 & 7 & 23 & 0 & 13 & 14 & 3 \\
\hline \multirow{2}{*}{$(0 \%$ vs. $5 \%)$} & 50 & 13 & 16 & 1 & 14 & 8 & 8 \\
\hline & 500 & 23 & 0 & 7 & 17 & 0 & 13 \\
\hline
\end{tabular}

Results are given for the 25th, 50th and 500th generations. A: Mean initial frequency of the favourable alleles in fitness was 0.5 . B: Mean initial frequency of the favourable alleles in fitness was 0.1 .

3 per cent) and ( 0 vs. 5 per cent), respectively (Table $2, \mathrm{~A})$. For $P=0.1$ (Table 2,B) the most frequently fixed allele was the 3 per cent allele. The difference is significant with ( 0 vs. 5 per cent) (chi-squared, 1 d.f. $=4.48)$ but not with ( 0 vs. 1 per cent) (chi-squared, 1 d.f. $=2.19$ ). The latter comparison should become significant when increasing the number of simulations.

In small populations ( $N=100$, Table 3$)$, no population remained polymorphic at the mating locus at the 500th generation. As in large populations for $P=0.5$, the 1 per cent allele is the most often fixed outcrossing allele (Table 3,A) but this trend is not significant with 30 replicates. For $P=0.1$ (Table $3 b$ ), the 5 per cent allele had the highest probability of being fixed. This probability was significantly lower for 1 per cent (chisquared, 1 d.f. $=5.08$ ) but not for 3 per cent (chi-squared, 1 d.f. $=1.83$ ). In small populations for $P=0.5$, fixation took place faster in the $(0$ vs. 1 per cent) case than for the other two patterns because 22 populations of 30 were fixed (either for selfing or outcrossing alleles) straight after 50 generations compared with 16 cases for ( 0 vs. 3 per cent) (n.s.) and 14 cases for $(0$ vs. 5 per cent $)$ (chi-squared, 1 d.f. $=4.44)$.

\section{Discussion}

\section{Optimal outcrossing rate for populations}

Under group selection, we found that mixed mating systems were optimum for a fast and achieved adapta- tion. In comparison strong selfing allowed a faster genetic advance in first generations but this advance becomes rapidly weak. Panmixia prevented the fixation of unfavourable alleles but gave quite a slow rate of genetic improvement.

When all individuals had the same mating behaviour, outcrossing and selfing accumulated their advantages to achieve an optimum genetic advance. The fitness increase from one generation to another was achieved with the available genotypic variance and through the way the offspring inherited the value of their parents.

Selfing allowed efficient selection. The parentoffspring and hence the heritability are higher in a selfing than in an outbreeding population when gene frequencies are identical. First, selfed progenies inherit the entire additive values of their parental line whereas the values of outcrossed progenies are diluted one-half of the whole population mean fitness. Secondly, in a highly polymorphic population in which there is no linkage disequilibrium (as in the first generations of our simulations), selfed progeny of a heterozygous individual are distributed with a larger variance than outcrossed progenies of the same individual. As there was no inbreeding depression, selfing then permitted the production of valuable individuals and the rapid increase in their frequency.

Outcrossing recreated genotypic variance. With finite populations, selfing and selection rapidly establish strong genetic disequilibrium between favourable and unfavourable alleles at different loci by select- 
ing only a few valuable genotypes (Bulmer, 1971). This efficient selection quickly depleted the genotypic variance, reduced the effective reproductive number and then impaired further genetic advance. Because of genetic drift, polymorphism should have vanished if no recombination took place. Under the optimum mixed mating system, cross-pollination recombined the genes of the most valuable genotypes. This recreated genotypic variance. Selection remained efficient even when genic disequilibria appeared. By preserving rare favourable alleles, outcrossing allowed a better management of variability for further improvement.

In our model with polygenic fitness, the respective advantages of outcrossing and selfing highly depended on the population size and the initial mean frequency of the favourable fitness alleles. Therefore, optimum mating systems were also dependent on these parameters. In very large populations, the optimum will lie near complete selfing. In an infinite initial population the perfect genotype, combining all the favourable alleles, already exists even if its frequency is very low. It will be quickly selected under selfing. This is not so in finite small populations, where the values of the best individuals are close to the population mean. In this case, genetic advance can be achieved only through selection and recombination. Similarly, chances to lose favourable alleles are higher when their frequencies are low and this happens in spite of the selection acting on them. Thus, the smaller is the population size and the weaker the frequency of favourable alleles, the higher the optimum outcrossing rates.

\section{Outcrossing under individual selection}

Under individual selection for mating behaviour, the intrinsic segregation advantage of selfing (Fisher, 1941) did not permit the selection of an optimum mating system for the population. Under neutrality, our results strictly followed the predictions of theoretical models (Fisher, 1941; Wells, 1979): selfing is the most selective behaviour and low outcrossing can only become fixed by random genetic drift in small populations. Furthermore, these alleles are counter-selected all the more rapidly as they give higher outcrossing rates (Wells, 1979). Nevertheless, their recombination advantage permitted outcrossing alleles to become fixed when populations evolved under strong directional selection. This can be explained with the previously mentioned mechanisms for group selection and individual selection under neutrality. During the first generations, the selfing allele was fixed faster. This comes from the absence of strong linkage disequilibrium between favourable and unfavourable alleles for that period and also from a sufficient amount of geno- typic variance to ensure population improvement under selfing. The segregation advantage of selfing prevailed over that of outcrossing. If by chance the outcrossing allele did not vanish during this first period, because of a large population size for example, it could begin to benefit by its recombination advantage. The valuable genotypes were then issued from out-pollination and they carried away the outcrossing allele by hitch-hiking. The interplay between selection, drift and hitch-hiking thus allowed the outcrossing allele to be fixed more often than under neutral conditions. The strength of the recombination advantage depends on complex interactions and varied according to the population parameters. In individual selection as in group selection, the smallest population sizes and lowest frequencies of favourable alleles gave a bigger advantage to the progenies of outcrossed plants than to the progenies of selfed plants. The genetic drift also led to fixation of strong outcrossing rates with higher probability in small populations. The interplay between selection and genetic drift explains the fact that the 1 per cent allele in the $(N=1000, P=0.5)$ populations showed the highest probability of fixation when it was the 5 per cent allele in the $(N=100$, $P=0.1)$ populations. Other alleles had a smaller selective value: when they were higher outcrosses, their intrinsic segregation handicap was too strong compared with the hitch-hiking effect they benefited from and when lower they could not counterbalance the loss of favourable genes by drift. This explains why they became fixed in fewer cases.

\section{Gene flows and hitch-hiking}

While gene flows were regular with a fixed mating system, favourable outcrossing events varied to a large extent under individual selection with regard to the outcrossing allele frequency. They were probably rare and proceeded from very few individuals. From a new transgressive heterozygous individual, good homozygous lines were derived because selfing remained the main mating way (from 95 per cent to 99 per cent). By chance the very best transgressive lines could be fixed either for outcrossing alleles or for selfing. Rapid selection of the best lines may then lead to rapid changes in outcrossing allele frequencies because of hitch-hiking. Owing to the finite size of populations, a favourable recombination event can thus paradoxically lead to the fixation of selfing. According to these recombination events, increases and decreases in mean outcrossing rate followed on (Fig. 4), making gene flows heterogeneous.

Under individual selection, the rapid selection of a few good lines quickly increased the relatedness within 


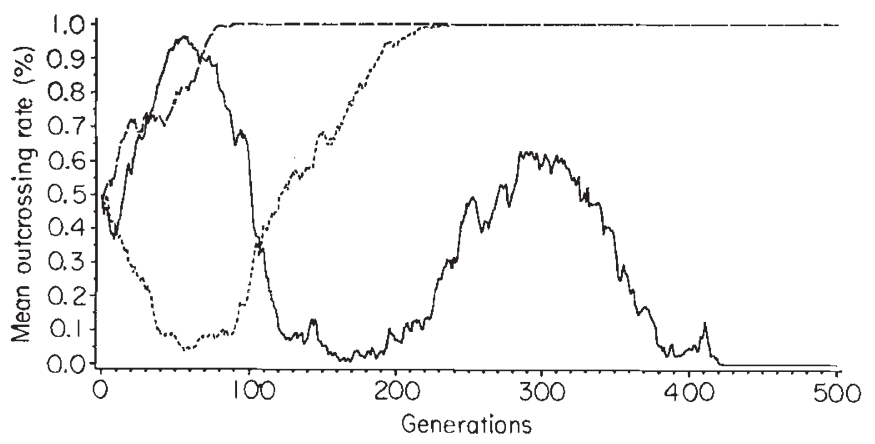

Fig. 4 Some patterns of evolution of the outcrossing rate under directional selection. Each curve represents the evolution of a single population of 1000 individuals with a starting frequency of the favourables alleles (at fitness locus) equal to 0.5 . The outcrossing rate is determined by a locus with two alleles, ' 0 ' and ' 0.005 ', so that homozygous genotypes ' $0.005^{\prime} / \times 0.005^{\prime}$ out-pollinate 1 per cent of their ovules, heterozygous genotypes ' $0.005 \%$ ' 0 ' out-pollinate 0.5 per cent of their ovules and ' 0 ' $/{ }^{\circ} 0$ ' individuals are complete selfers.

populations. Genes were finally exchanged between related lines and recombination lost its interest and the segregation advantage of the selfing allele recovered some strength. The genetic advance realized under individual selection was consequently found to be much lower than under group selection. The intrinsic advantage of selfing prevented the group from attaining an optimum adaptation. The population as a whole showed less interest than its component individuals in mating. Similar results have been found by Lloyd (1979).

When the genetic variability became exhausted, natural evolution led to the loss of outcrossing alleles if they were not yet fixed at this stage. Moreover, complete selfing would be the only stable state after a longterm evolution if new selfing alleles could continuously appear by mutation. On the other hand, new mutating outcrossing alleles could be selected after fixation of selfing if population fitness were to remain far from its optimum or if deleterious mutations were to reduce the adaptation level of the population. If so, our model would be very close to recent theoretical developments for the evolution of mating. These models introduce possibilities of stable mixed mating systems, for instance when inbreeding depression increases exponentially with generations (Damgaard $e t$ al., 1992) or when selfing and outcrossing enhancers develop positive associations with favourable viability alleles when deleterious genes continuously appear by mutation (Campbell, 1986; Holsinger, 1988; Uyenoyama \& Waller, 1991).

\section{Conclusion}

As our model did not require any assumption on inbreeding depression or ecological factors, it differed from almost all the models dealing with the evolution of mating systems through inbreeding depression and 'cost of outcrossing' (Maynard-Smith, 1978; Charlesworth, 1980; Feldman \& Christiansen, 1984). We simulated populations evolving under strong directional selection for fitness considered as a quantitative trait and not affected by inbreeding depression. We showed that the optimum advance in fitness was realized with mixed mating systems under group selection. We also pointed out that maintenance of low levels of outcrossing was possible under individual selection in finite heterogeneous populations, at least for long transient periods and especially when populations were small and ill-adapted to their environment.

The advantage of outcrossing is to be discussed according to the results found for the advantage of recombination under selection (Lewontin, 1974; Crow, 1992). Maynard-Smith (1978) discusses it for complex unpredictable ecosystems. Biologically complex and changing environments continuously move the optimum fitness with qualitatively new unpredictable forces, such as new pathogens' spectrum or competitors for resources. From a dynamic point of view, these results are well described by the Red Queen's theory (van Valen, 1973): favourable genes have to recombine so as not to be lost with time. Some experimental results obtained on barley could also be partially explained by our model. Kahler et al. (1975) showed that outcrossing rate in heterogeneous barley populations, obtained by combining genes of very different parental lines, increased twofold (from $t=0.006$ to $t=0.012$ ) after 28 generations of natural selection whereas during the same time many evolutions were observed on morphological traits and allelic frequencies of esterase loci. Outcrossing seems to have been implicated in creating new adapted lines (Allard, 1979), and in turn it could have been hitch-hiked.

The maintenance of low outcrossing in a network of populations of a predominantly selfing species would be possible if it evolved in disturbed conditions and if individuals migrated from one site to another. In such a metapopulation (Levins, 1970), different levels of outcrossing could be found in populations according to their site, their age and their size as it has been proposed for migration abilities (Olivieri \& Gouyon, 1985). Group selection could occur between subpopulations of a metapopulation. This would result in an increase of the mean outcrossing rate as we demonstrated that fitness increased faster under a quite high optimal outcrossing rate. 


\section{Acknowledgements}

The authors are grateful to Dr Couvet for scientific comments and proposals, to Muriel Guittet and Guy Decoux for computer advice and to Evelyne Tuck for carefully reading the manuscript.

\section{References}

AGREN, J. AND SCHEMSKE, D. W. 1993. Outcrossing rate and inbreeding depression in two annual monoecious herbs, Begonia hirsuta and Begonia semiovata. Evolution, 45, 125-135.

ALLARD, R. W. 1979. The mating system and microevolution. Genetics, 79, 115-126.

Allard, R. W. AND wORKMAN, P. L. 1963. Population studies in predominantly self-pollinated species. IV. Seasonal fluctuation in estimated values of genetic parameters in lima bean. Evolution, 17, 470-480.

BULMER, M. G. 1971. The effect of selection on genetic variability. Am. Nature, 105, 201-211.

CAMPBELL, R. B. 1986. The interdependence of mating structure and inbreeding depression. Theor. Pop. Biol., 30, 232-244.

CHARLESWORTH, B. 1980. The cost of sex in relation to mating system. J. Theor. Biol., 84, 655-671.

CHARLESWORTH, D., MORGAN, M. T. AND CHARLESWORTH, B. 1990. Inbreeding depression, genetic load and the evolution of outcrossing rates in a multilocus system with no linkage. Evolution, 44, 1469-1489.

CROW, J. F. 1992. An advantage of sexual reproduction in a rapidly changing environment. J. Heredity, 83, 169-173.

DAMGAARD, C., COUVET, D. AND LOESCHKE, v. 1992. Partial selfing and optimal mating strategy. Heredity, 69, 289-295.

FELDMAN, M. V. AND CHRISTIANSEN, F. B. 1984. Population genetic theory of the cost of inbreeding. Am. Nature, 123, $642-653$.

FISHER, R. A. 1941. Average excess and average effect of a gene substitution. Ann. Eugen., 11, 53-63.

HARDING, J. AND TUCKER, C. L. 1965. Quantitative studies on mating systems. I. Evidence of non-randomness of outcrossing in Phaseolus lunatus. Heredity, 19, 369-381.

HOLSINGER, K. E. 1988. Inbreeding depression doesn't matter, the genetic basis of mating system evolution. Evolution, 42, 1235-1244.
Hospital, F. AND CHEVAleT, c. Effect of population size and linkage on optimal selection intensity. Theor. Appl. Genet. (in press)

KAHLER, A. L., ClegG, M. T. AND Allard, R. W. 1975. Evolutionary changes in the mating system of an experimental barley population (Hordeum vulgare L.). Proc. Natl. Acad. Sci. U.S.A., 72, 943-946.

LANDE, R. AND SCHEMSKE, D. W. 1985. The evolution of self-fertilization and inbreeding depression in plants. I. Genetic models. Evolution, 39, 24-40.

LÉCUYER, P. 1988. Efficient and portable combined random number generators. Communications of the ACM., 31, 742-774.

LEVINS, R. 1970. Extinction. In: Providence, R. I. and Gesternhaber, M. (eds), Some Mathematical Questions in Biology, American Mathematical Society, pp. 77-107.

LEwontin, R. C. 1974. The Genetic Basis of Evolutionary Change. Columbia University Press.

LLOYD, D. G. 1979. Some reproductive factors affecting the selection of self-fertilization in plants. Am. Nature, 113 , 67-79.

MANI, G. S., CLARKE, B. C., SHELTON, F. R. S. AND SHELTON, P. R. 1990. A model of quantitative trait under frequency dependent balancing selection. Proc. R. Soc. Lond., 240, 15-28.

MAYNARD-Smith, J. 1978. The Evolution of Sex. Cambridge University Press, Cambridge.

NAGYLAKI, T. 1976. A model for the evolution of self-fertilization and vegetative reproduction. J. Theor. Biol., 58, 55-58.

OLIVIERI, I. AND GOUYON, P. H. 1985. Seed dimorphism for dispersal: theory and implications. In: Haeck, J. and Woldendorp, J. W. (eds), Structure and Functioning of Plant Populations, North Holland Publishing, Amsterdam, pp. 77-90.

SCHEMSKE, D. W. AND LANDE, R. 1985. The evolution of selffertilization and inbreeding depression in plants. II. Empirical observations. Evolution, 39, 41-52.

UYENOYAMA, M. K. AND WALLER, D. M. L. 1991. Coevolution of self-fertilization and inbreeding depression. I. Mutationselection balance at one and two loci. Theor. Pop. Biol., 40, 14-46.

van valen, L. 1973. A new evolutionary law. Evol. Theor., 1, $1-30$.

WELLS, H. 1979. Self-fertilization, advantageous or deleterious? Evolution, 33, 252-255. 